\title{
Extraction of Virtual Scattering Centers of Vehicles by Ray-Tracing Simulations
}

\author{
Karin Schuler, Denis Becker, and Werner Wiesbeck, Fellow, IEEE
}

\begin{abstract}
Radar images of complex targets can be understood as a superposition of the reflected signals from a high number of scattering centers. To model complex targets for radar simulations, the plurality of scattering centers should be reduced to few significant scattering centers in order to minimize computational effort. The scope of this work is to present a technique to generate a significantly simplified RCS model of the vehicle with a limited number of virtual scattering centers, each with its own scattering characteristic, and how to group these scattering centers in a cluster database. The work is based on ray-tracing simulations of complex vehicle models. The ray-tracing simulations have been validated by measurements. The scattering centers may not be physically existing strong scattering centers, but virtual scattering centers representing a certain scattering behavior. In this paper, a technique for extracting such virtual scattering centers from a complex 3D-vehicle-model is presented. It is based on ray-tracing simulations of such models. As an example, the design model of a Ford Focus is used.
\end{abstract}

Index Terms-Ray-tracing, RCS-modeling, scattering center.

\section{INTRODUCTION}

D URING the last years, safety relevant sensor systems have become an important feature in the automotive industry. Presently, short range radar (SRR) systems are being developed and introduced to the market. Their intention is to cover the near surrounding of a vehicle to assist the driver during Stop-n-Go traffic and parking, but also to increase safety by blind-spot surveillance and side impact warnings. Currently, different approaches are being discussed for the realization of a radar sensor with full azimuth coverage and high azimuth resolution of the close-by environment. To simulate and evaluate the performance of different approaches for such SRR-systems, scattering-models of vehicles are required. Since vehicles observed in the close-by region exhibit multiple scattering centers, contributions from different angles are expected. Depending on the incidence angle, they cause multiple intensity maxima in radar images [1]. By describing the scattering characteristics of complex objects by one single RCS value, the multiple scattering centers and other scattering phenomena are not apparent

Manuscript received January 22, 2007; revised July 12, 2008. Current version published November 14, 2008.

K. Schuler was with the Universität Karlsruhe (TH), Institut für Höchstfrequenztechnik und Eletronik (IHE), Karlsruhe D 76131, Germany. She is now with EADS Defence Electronics, Ulm D 89077, Germany (e-mail: karin.schuler@ihe.uka.de).

D. Becker and W. Wiesbeck are with the Universität Karlsruhe (TH), Institut für Höchstfrequenztechnik und Eletronik (IHE), Karlsruhe D 76131, Germany (e-mail: denis.becker@ihe.uka.de; werner.wiesbeck@ihe.uka.de).

Color versions of one or more of the figures in this paper are available at http://ieeexplore.ieee.org.

Digital Object Identifier 10.1109/TAP.2008.2005436 anymore. For this reason, it is not sufficient to consider a single scattering center or an azimuth independent RCS in the simulations. However, the multiple scattering centers are important and have to be taken into account in radar imaging simulations with high azimuth resolution. A scattering center description for target recognition in one dimension is proposed in [2]. Approaches for scattering center extraction are suggested in [3], [4].

There exist various numerical methods for the calculation of electromagnetic field distributions like the method of moments (MoM) [5], finite-difference-time-domain (FDTD) method [6] and the finite element method (FEM). All these methods require a high discretization of the structure relative to the wavelength. At high frequencies, this leads to an immense computational effort for large structures. Therefore, these numerical methods are not suited for large problems [7]. In such cases, hybrid [8] or asymptotic methods based on geometrical optics (GO) or physical optics (PO) are often used. Physical optics may also be extended by physical theory of diffraction (PTD) [7], the method of equivalent currents (MEC) [9] and impedance boundary condition (IBC) techniques [10].

These ray-tracing simulations deliver fast and reliable results when considering the scattering characteristic of complex objects. However, sometimes even these simulations of complex models are too time-consuming. For electrically large problems like traffic scenarios, it is therefore crucial to derive a simplified scattering model, which delivers a good approximation for the scattering characteristic of the involved vehicles.

In the following, a straightforward approach to generate such a simplified scattering model by determining the virtual scattering centers of the vehicle directly from ray-tracing simulations will be presented.

In general, ray-tracing simulations determine the properties of the propagation paths between a transmitter and a receiver, including multiple non-line-of-sight paths. On the way from the transmitter to the receiver, the rays hit the simulation structure at multiple points, which leads to reflection, diffraction and scattering. All these interaction points are called scattering centers. The scattering centers are therewith a direct result of the ray-tracing simulation. The goal of this work is to group the scattering centers into so-called virtual scattering centers. The virtual scattering centers are representatives of the scattering behavior of the structure itself and describe the most important geometrical parts and their contributions to the scattering.

The ray-tracing simulation results have been validated by measurements. Based on these results, a simplified scattering model is derived, consisting of multiple virtual scattering centers, each with its special scattering characteristic. These virtual scattering centers represent the simplified scattering 


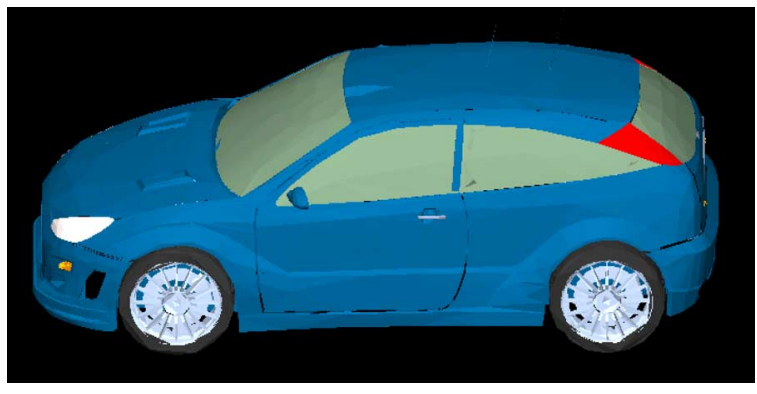

Fig. 1. Model of Ford Focus for IHE 3D-ray-tracing.

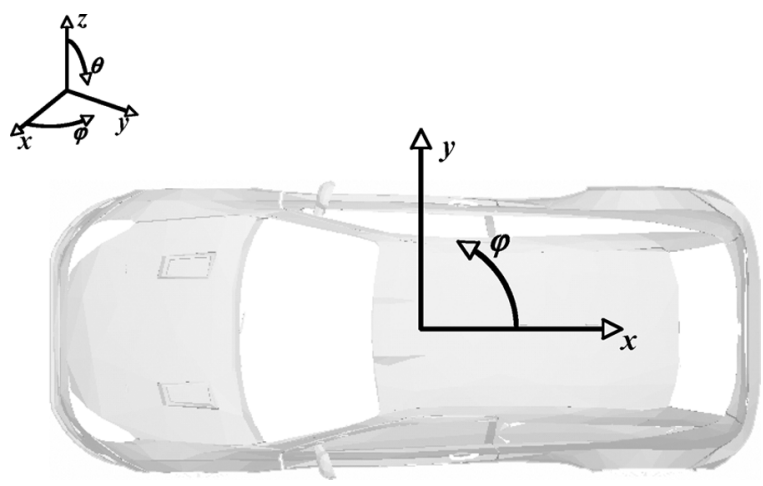

Fig. 2. Coordinate system.

model, which has a similar scattering behavior as the complex car model. This simplified scattering model allows the rapid investigation of a wide range of radar scattering situations, where a conventional ray-tracing method would be too complex or is not available.

\section{RAY-TRACING SiMULATION}

Simulations of vehicles with commercial FDTD simulation tools are not feasible at frequencies of $24 \mathrm{GHz}$ or $76 \mathrm{GHz}$, as they are specified for automotive radar applications. The discretization of the model into sub-wavelength-elements would lead to too large matrices. Ray-tracing simulations do not suffer directly from this constraint. The crucial number for ray-tracingsimulations is the number of visible faces seen by the transmitter. This number is therewith rather related to the geometry's complexity than to its size. RCS simulations with ray-tracing have also been presented in [7].

One approach for the determination of scattering centers is presented in [11]. In simulated ISAR images intensity maximums are considered as scattering centers and subtracted from the radar image with the clean algorithm [12]. The radar images are based on the shooting and bouncing ray technique and on the processing of ISAR images. This procedure therefore requires a detour when processing the ISAR image.

A similar but frequency and aspect dependent technique for data compression of SAR and ISAR images is presented in [13]. It is based on the extraction of point and line-segment scatterers from the measured radar image.

In the following, the scattering information obtained by the ray-tracing simulation will be directly evaluated. The used ray-

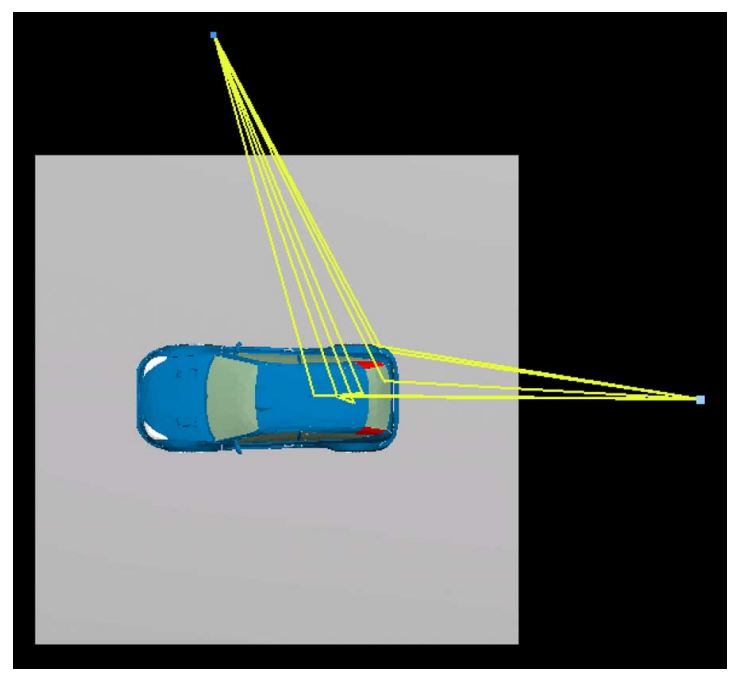

Fig. 3. Example of bistatic ray-tracing simulation with IHE 3D-ray-tracing.

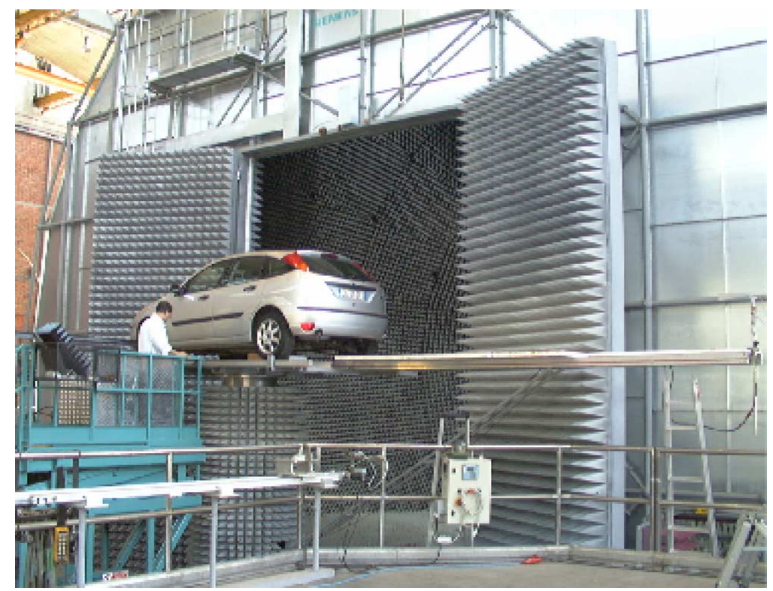

Fig. 4. Bistatic measurements at JRC, Ispra, Italy, car outside the chamber.

tracing tool has been developed at the Institut für Höchstfrequenztechnik und Elektronik (IHE) at the University of Karlsruhe (TH) in Germany. It is a ray optical approach for modeling wave propagation. Each ray is considered separately and takes into account multiple reflections, diffraction and scattering. This gives an insight to the scattering centers and the scattering phenomena that will be exploited in the following. Modified Fresnel coefficients are used to model rough surfaces. Diffraction is described by the uniform geometrical theory of diffraction (UTD) and the corresponding heuristic coefficients for wedge diffraction. The ray-tracing tool has already been verified multiple times with measurements for various wave propagation simulations [14]-[17].

The 3D-model of the car, which is considered in the following, is illustrated in Fig. 1.

The model of the car consists of 12.100 triangles. Each of them is at least $25 \mathrm{~cm}^{2}$, guaranteeing a large area compared to the wavelength at $f=24.125 \mathrm{GHz}(\lambda=1.19 \mathrm{~cm})$. This is important to fulfill the requirements of the ray-tracing tool. For calculating the reflections, the ray-tracing tool assumes relatively large areas and uses the modified Fresnel-coefficients for the calculation of the reflection coefficient. Therefore, the edge 

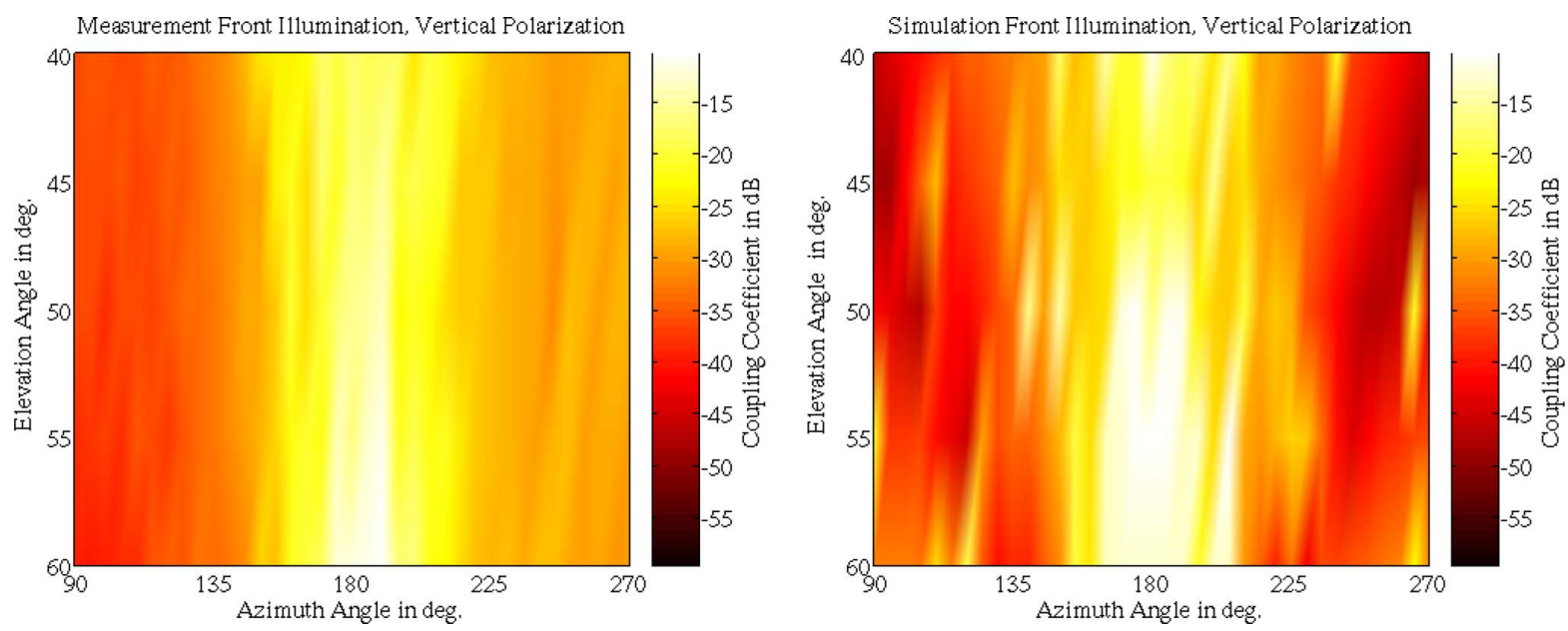

Fig. 5. Bistatic coupling coefficient for front illumination $\left(\varphi=180^{\circ}\right)$ in vertical polarization at $f=24 \mathrm{GHz}$. Left: measurement, right: simulation.
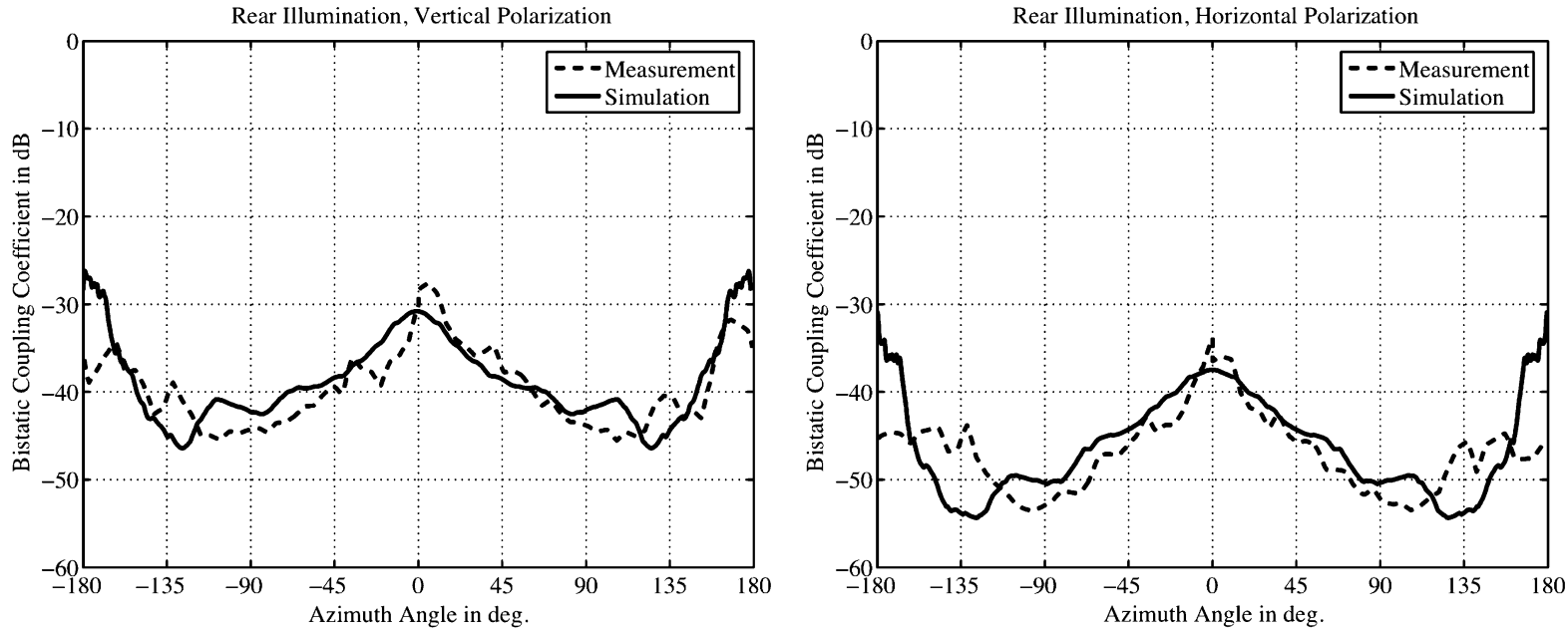

Fig. 6. Bistatic coupling coefficient at elevation angle $\theta=60^{\circ}$ for rear illumination $\left(\varphi=0^{\circ}\right)$. Left: vertical polarization, right: horizontal polarization.

length of an element must be within 5 to 10 wavelength, which is fulfilled in this case. This condition determines also the maximum number of elements. In this particular case of a vehicle, reflections are the majority of all interactions. If the number of elements is chosen significantly smaller, the ray-tracer will not find enough reflections to represent the scattering characteristic of the car precisely enough. This would also be the case for lower frequencies, for which the size of the triangles would have to be increased and therefore their number would become to small to give a precise representation of the cars geometry. For higher frequencies, more detailed models could be used, delivering even more accurate results. A material assignment is made for each discretization element of the car. It contains the parameters of the permittivity, the roughness and the loss. For the simulation, a ground floor was added to take multipath propagation effects also into account [18].

\section{VERIFICATION OF RAY-Tracing SimUlations}

The bistatic scattering coefficient of a Ford Focus has been simulated with ray-tracing and compared to measurements of the same vehicle. In Fig. 2 the coordinate system and its origin is defined relative to the car.

Fig. 3 shows the top view for a bistatic ray-tracing simulation. The lines indicate the propagation paths.
The line-of-sight path is neglected. For each transmitter and receiver position, the dynamic range is limited to $100 \mathrm{~dB}$ with reference to the strongest non-line-of-sight path. Up to five interactions as diffraction and reflection are considered per path. For scattering only one interaction is considered, since the power level of the scattered path is reduced drastically. For each path, the path information is stored. The path information contains the number, types and locations of the interactions. Also the amplitude and the phase of the received signals are delivered. These parameters are obtained from the propagation time and the complex scattering coefficients of the interactions.

The vehicle is illuminated from the rear $\left(\varphi=0^{\circ}\right)$ in the azimuth plane $\left(\theta=90^{\circ}\right)$ whereas the receiver performs a $360^{\circ}$-turn along the azimuth. The simulations have been performed for both horizontal and vertical polarization. Similar simulations were performed for front illumination $\left(\varphi=180^{\circ}\right)$. The same configurations are used in the verification measurements.

The verification measurements were performed in the anechoic chamber of the European Commission Joint Research Center (JRC), located at Ispra in northern Italy, and were published in [19]. Fig. 4 shows the vehicle in front of the anechoic chamber. 
With this setup, the bistatic coupling coefficient along the azimuth angle $\varphi$ and the elevation angle $\theta$ has been measured for the two cases: front and rear illumination, both in vertical and horizontal polarization. The coupling coefficient for front illumination has been simulated and measured along a spherical surface. It is plotted along the azimuth angle $\varphi$ and the elevation angle $\theta$. In Fig. 5 the comparison between measurement and simulation is shown for vertical polarization. The transmit antenna was placed at azimuth angle $\varphi=180^{\circ}$ and elevation angle $\theta=90^{\circ}$. The receive antenna was moved along azimuth from $\varphi=90^{\circ}$ to $\varphi=270^{\circ}$ and along elevation from $\theta=40^{\circ}$ to $\theta=60^{\circ}$ in the upper hemisphere. Measurements have already been performed for the study presented in [19] with an angular step width of $5^{\circ}$ along azimuth and elevation. For the comparison between the measurement and the simulation, the bistatic simulations have been performed with the same step width.

The comparisons show a good agreement for high elevation angles $\left(\theta \approx 60^{\circ}\right)$, which are close to the azimuth plane. The farther transmitter and receiver are positioned from each other, the more simulation and measurement differ from each other. However, the closer transmitter and receiver are positioned to each other, the better is the agreement. Since in the following only monostatic simulations will be analyzed, reliable results from these ray-tracing simulations are expected.

For a closer look at the data, the coupling coefficient for a constant elevation angle $\theta=60^{\circ}$ is shown in Fig. 6. It shows the comparison of the simulated and the measured coupling coefficient for rear illumination in vertical (left) and horizontal (right) polarization. For both polarizations, the coupling coefficient drops significantly when transmit and receive antennas are oriented $\pm 90^{\circ}$ relative to each other. The agreement between measurement and simulation is very good for azimuth angle $\varphi$ between $-160^{\circ}$ and $160^{\circ}$. For azimuth angles close to $\varphi=180^{\circ}$, the angle of reflection is relatively large. In this specific configuration, the simulation leads to an increased reflection coefficient since the coupling along the roof of the car is considered to strong.

The here presented comparison uses a bistatic configuration due to the available measurements. It shows, that the closer transmit and receive antenna are situated to each other, the better is the agreement between simulation and measurement. This is important since the following simulations and the derivation of the virtual scattering model will be performed in monostatic configuration. This monostatic configuration can be looked at as the extreme case of a bistatic configuration, where both antennas are placed at the same location. However, the accuracy of the virtual scattering center model can not be higher than the accuracy of the initial ray-tracing simulation. This comparison proves the reliability of the ray-tracing simulations with this detailed simulation model of the Ford Focus. Therefore the analysis of ray-tracing specific information like the location of scattering centers and the path information can be used for further analysis.

\section{Monostatic Virtual Scattering Center Extraction}

In the following, monostatic ray-tracing simulations in azimuth will be evaluated. The analysis of the scattering centers

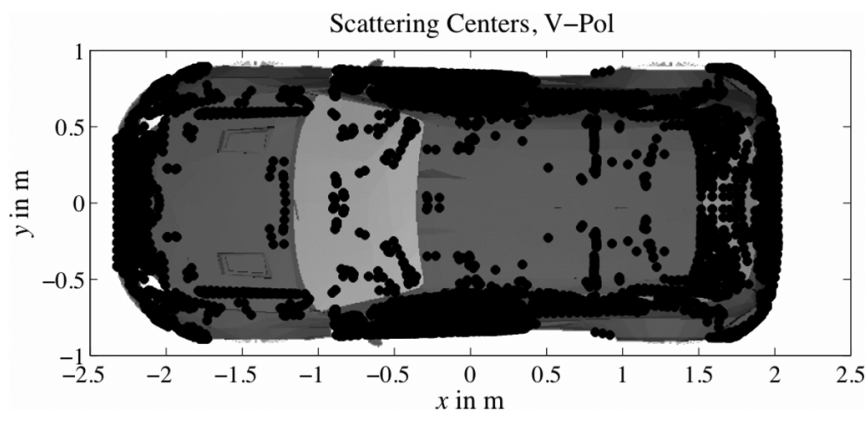

Fig. 7. Scattering centers for monostatic simulation of Ford Focus.

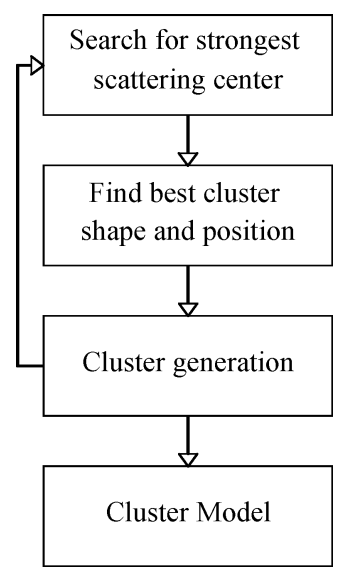

Fig. 8. Flowchart of implemented greedy-algorithm.

leads to scattering clusters. To each cluster, a single virtual scattering center with its own scattering characteristic is assigned. The scattering characteristic is obtained by summarizing the scattering effects of all scattering centers belonging to one cluster. Of course, a high number of clusters will lead to a more precise model, but on the other hand, a model containing a low number of clusters will result in a faster calculation. It will be shown that it is possible to reduce the complex vehicle model to a limited number of clusters for characterizing the scattering behavior in the azimuth plane without compromising on the precision of the simulation.

\section{A. Simulation of Scattering Centers}

A monostatic simulation of the reflection coefficient has been performed along azimuth using $1^{\circ}$ step width along a circle with the radius $r=3 \mathrm{~m}$. Regarding the possible application of this model for Short Range Radar simulations, the angular resolution width is a good compromise between computation time and accuracy. The position and the amplitude of the scattering centers depend on the radius and the azimuth angle during ray-tracing simulation. This fact has to be taken into account when the derived virtual scattering center model will be used in other radar simulations by calculating the aspect angles for each cluster and the attenuation.

In Fig. 7, the scattering centers for a monostatic simulation in vertical polarization for elevation angle $\theta=90^{\circ}$ and azimuth angle varying from $\varphi=0^{\circ}$ to $\varphi=360^{\circ}$ are plotted onto the car 

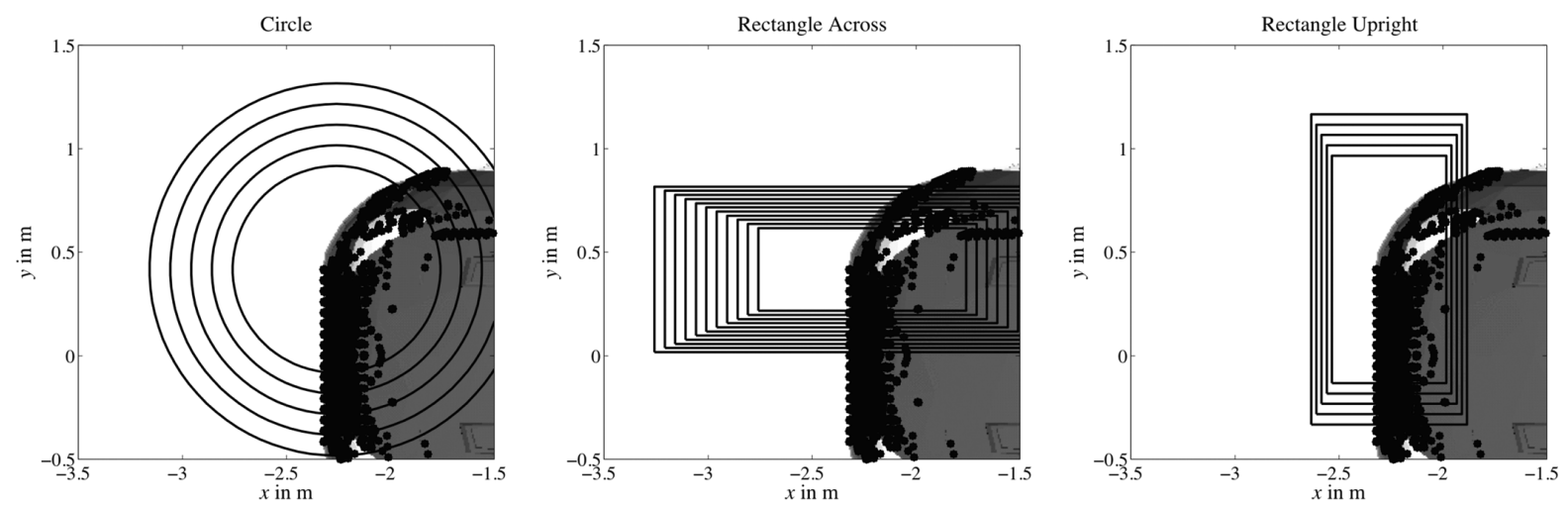

Fig. 9. Adaptive clustering for size and shape. Left: circle middle: rectangle across. Right: rectangle upright.

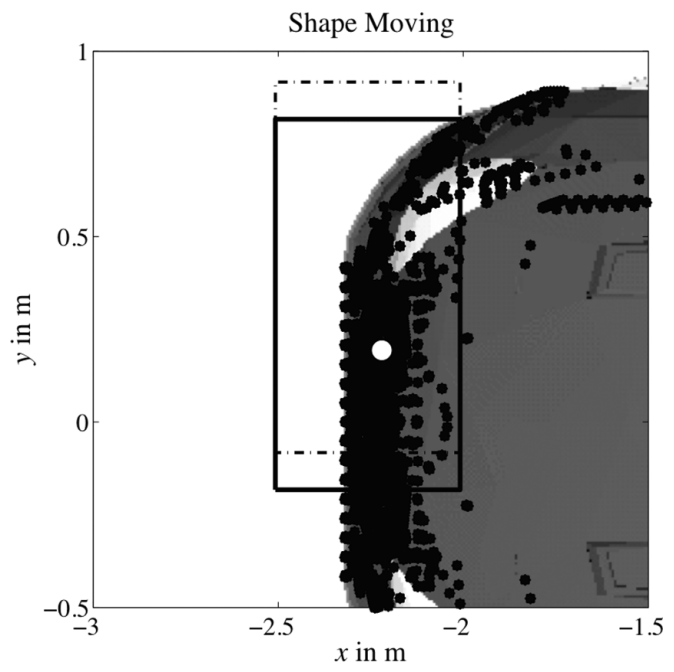

Fig. 10. Shape moving of original position (dashed) to find optimum fit position (solid).

as black dots. Each scattering center is active for a certain direction with a specific amplitude and phase. This diagram illustrates the important parts of the vehicle regarding the scattering in general. For each specific direction, only a part of the shown scattering centers is contributing to the scattering characteristic. In total, all scattering centers lead to the typical scattering characteristic, which is taken later on as a reference.

\section{B. Extraction of Scattering Clusters and Virtual Scattering Centers}

To simplify the model, the scattering centers are arranged into scattering clusters. This is done by a so called greedy-algorithm [20]. In the first part the scattering centers are arranged by their location and in the second part the contribution of the scattering center into a certain direction is considered.

In the following the greedy algorithm, which is illustrated in the flowchart in Fig. 8, is described in detail.

Search for Strongest Scattering Center: In the first step, the algorithm looks for the scattering center related to the strongest scattering center. This is the starting point for the cluster formation. In the surrounding of this point, the algorithm then searches for further scattering centers.

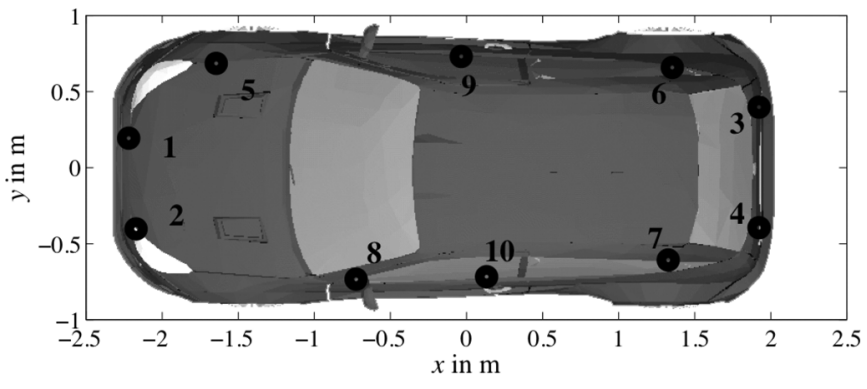

Fig. 11. Virtual scattering centers of Ford Focus.

Find Best Cluster Shape and Position: Adaptively, the algorithm decides on the shape of the cluster. The cluster shape and size affect the cluster extraction. If the cluster shape and the geometrical structure of the scattering centers do not match, the algorithm will determine a high number of clusters for an appropriate description. The cluster size also affects the number of determined clusters. If the cluster size is to small, a higher number of clusters is required to take into account all contributions from the scattering centers. On the other hand, large clusters sum up the contributions from a large number of scattering centers. This reduces the effect of multiple scattering centers. In the extreme case, when all scattering centers are combined into one cluster, the conventional radar cross section is obtained, which cannot be used for simulations evaluating multiple scattering centers.

In the case of the presented vehicle, three different cluster shapes are considered: Circles, upright rectangles and across placed rectangles. These shapes have been chosen according to the top view geometry of the vehicle, which is basically a rectangle with rounded edges. The circle shapes have a radius $r$ from $r_{\min }=0.5 \mathrm{~m}$ to $r_{\max }=0.9 \mathrm{~m}$ to fit to the car edges. The aspect ratio of upright rectangles width $w_{\text {up }}$ to height $h_{\text {up }}$ is $w_{\text {up }} / h_{\text {up }}=0.5$. This is chosen due to the fact that the upright rectangles are intended to cover the bumpers. For the upright rectangles the height $h_{\mathrm{up}}$ is varied from $h_{\mathrm{up}, \min }=1.0 \mathrm{~m}$ to $h_{\mathrm{up}, \max }=1.5 \mathrm{~m}$. These values have been chosen relative to the car width of $w_{\text {car }}=1.9 \mathrm{~m}$, which is more than the maximum rectangle height $h_{\mathrm{up}, \max }$, but less than two times the minimum height $h_{\mathrm{up}, \min }$. For the rectangles across the aspect ratio $w_{\mathrm{acr}} / h_{\mathrm{acr}}=0.4$, which is the aspect ratio of the car itself, the width $w_{\text {acr }}$ is varied from $w_{\text {acr,min }}=1.0 \mathrm{~m}$ to $w_{\text {acr,max }}=2.0 \mathrm{~m}$. These values have been chosen relative to 

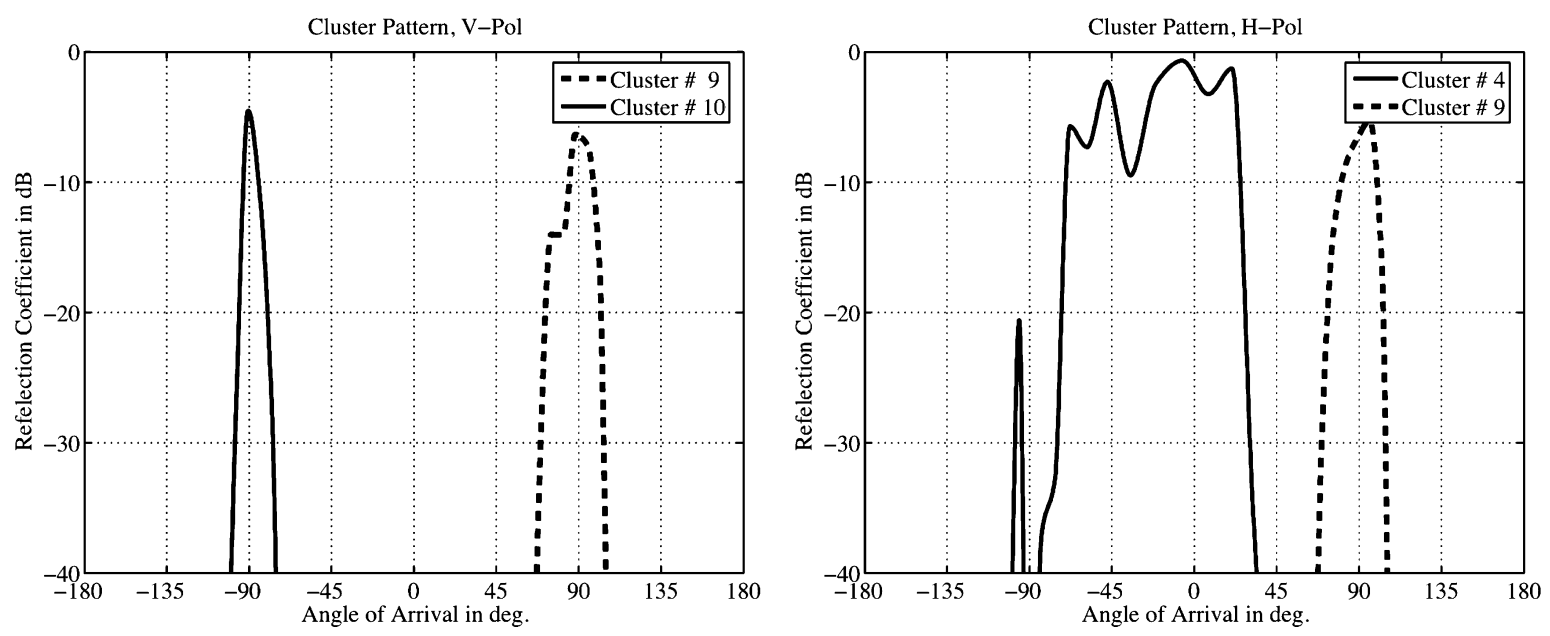

Fig. 12. Monostatic scattering characteristic for virtual scattering centers relative to their center. Left: cluster \#9 and cluster \#10, vertical polarization, right: cluster \#4 and cluster \#9, horizontal polarization.

the car length of $l_{\text {car }}=4.35 \mathrm{~m}$ to allow two to three clusters along the side of the car. These parameters are the only geometrical input parameters affecting the cluster search.

To achieve the maximum density of scattering centers per cluster area, the geometrical size of the cluster is varied within the given margins. This step of the algorithm is illustrated in Fig. 9.

Each cluster shape and cluster size is also moved in the $x-$ $y$-plane by $\delta x=0.3 \mathrm{~m}$ along $x$ and $\delta y=0.1 \mathrm{~m}$ along $y$ to achieve an optimum fitting of the shape to the location of the scattering centers. The initial position (dashed line) and the optimum fit position (solid line) are shown in Fig. 10 for a cluster at the right front of the car.

Cluster Generation: After a cluster has been determined, all scattering centers contained in that cluster are neglected during the determination of the next clusters. Hence the algorithm is called a greedy algorithm. To each generated cluster $c$, a virtual scattering center $\vec{x}_{C, c}$ is assigned. This is not the geometrical center of the cluster area, but the center of all $N_{S}$ scattering centers $\vec{x}_{s}(s)$ contributing to the cluster $c$, weighted by their reflectivity $D(s)$

$$
\vec{x}_{C, c}=\sum_{S=1}^{N_{S}} \vec{x}_{S}(s) D(s) .
$$

Also, for each cluster the monostatic scattering characteristic $C_{C, c}(\varphi)$ is calculated. For this, all complex contributions $A(s)$ of the $N_{S}(\varphi)$ scattering centers within the cluster contributing to the angle $\varphi$ are referred to the cluster center by the term $\mathrm{e}^{j \varphi(s)}$ and summed up

$$
C_{C, c}(\varphi)=\sum_{s=1}^{N_{S}(\varphi)} A(s) e^{j \varphi(s)} .
$$

Restart Search: After the determination of one cluster, the search for the next strongest signal restarts. This procedure is repeated until the contribution of the remaining scattering centers is below $-100 \mathrm{~dB}$ with reference to the strongest scattering center.

Fig. 11 shows the 10 virtual scattering centers determined by the algorithm for vertical and horizontal polarization. The numbers indicate the order in which the clusters are found by the algorithm. They are also ordered according to the reflection of the strongest scattering center within the clusters.

The strongest virtual scattering centers are at the four corners on the car, followed by the virtual scattering centers on wheelhouses. The positions of the virtual scattering centers are not symmetric. This is due to the fact that the implemented algorithm searches for the cluster centers one after the other without any pre-knowledge about the structure itself and its symmetry.

In the second step of the implemented algorithm, the scattering characteristic of the virtual scattering center is calculated. The contributions of all scattering centers within one cluster are referred to the virtual scattering center of the cluster and called reflection coefficient or scattering characteristic. For each polarization, the ray-tracing data is therefore evaluated. In Fig. 12 two scattering characteristics are shown for two individual virtual scattering centers. The angle of arrival AoA is relative to the virtual scattering center of each cluster.

In Fig. 12 left, the cluster characteristics are shown for cluster $\# 9$ and \#10 in vertical polarization. These are the sides of the car. It shows a strong influence for angles of arrival around $\mathrm{AoA} \approx \pm 90^{\circ}$. This means, that the clusters are active for incidence angles perpendicular to the side of the car, what one would expect. In Fig. 12 right, the cluster characteristic for cluster \#4 and \#9, the rear left cluster and a cluster at the right side of the car, are shown in horizontal polarization. Cluster \#9 has in horizontal polarization a similar influence as in vertical polarization. The influence of cluster \#4 is significant for angles of arrival from $\mathrm{AoA}=-70^{\circ}$ to $\mathrm{AoA}=25^{\circ}$. This is a relatively large angular coverage but since the cluster \#4 represents an edge of the car, this is evident.

Both examples show a high reflection coefficient for those aspect angles, where the clusters are visible. This indicates a reasonable description of the scattering characteristic. 

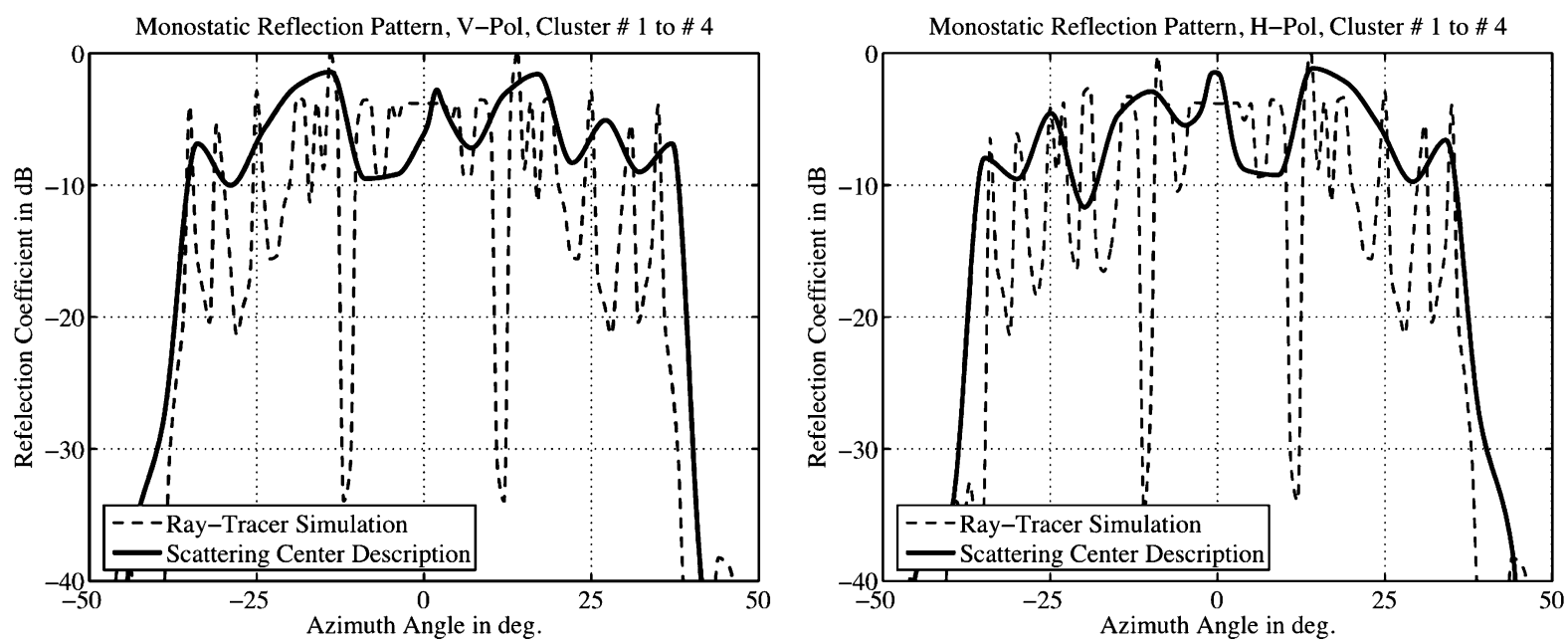

Fig. 13. Virtual scattering center description and ray-tracing simulation. Cluster \#1 to \#4 considered for virtual scattering center description. Left: vertical polarization, right: horizontal polarization.
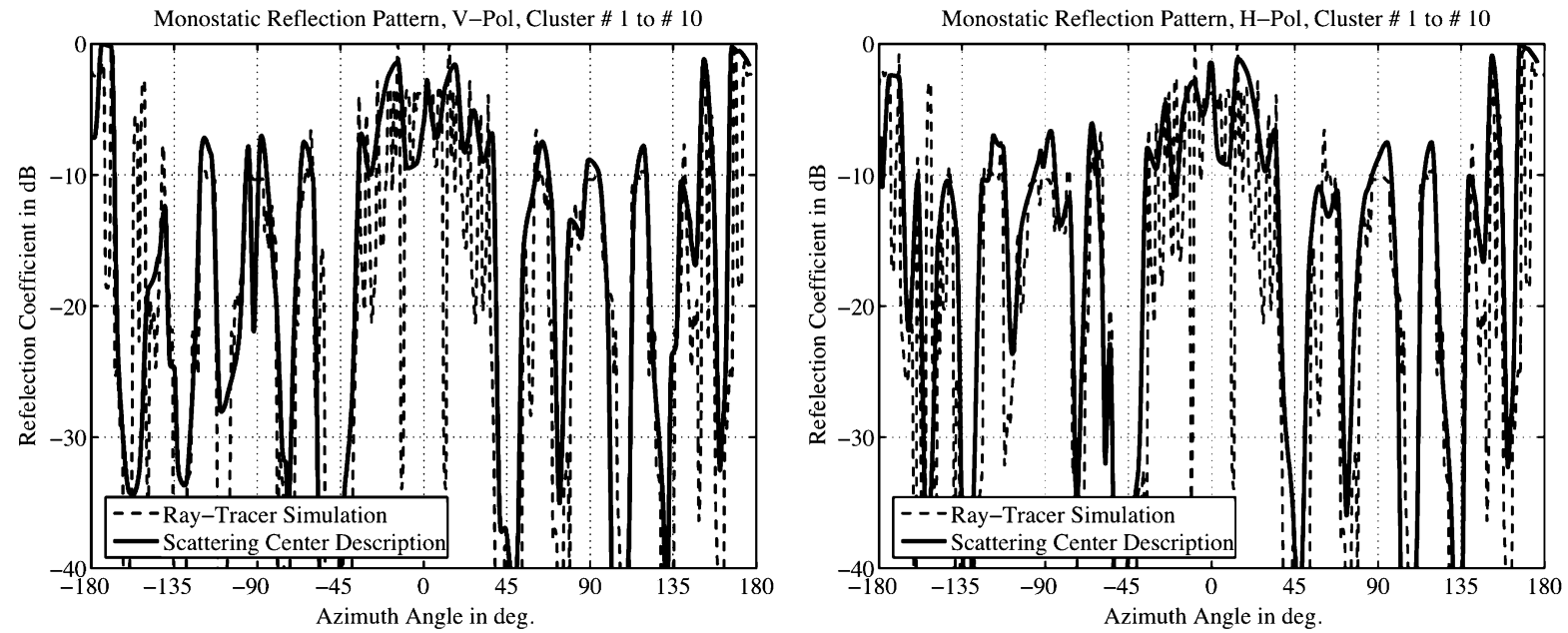

Fig. 14. Virtual scattering center description and ray-tracing simulation. Cluster \#1 to \#10 considered for virtual scattering center description. Left: vertical polarization, right: horizontal polarization.

\section{Validation of Virtual Scattering Center Description}

To validate the simplified car model of the Ford Focus, represented by the virtual scattering centers, its monostatic reflection coefficient is calculated. This is the summation over all scattering characteristics of the virtual scattering centers. For this, the scattering characteristics have to be referred to the origin of the coordinate system. This is a comparison between two simulations: The result of the original ray-tracing simulation and the corresponding virtual scattering center model. Both the ray-tracing simulation and the virtual scattering model might be different for other types of vehicles but a general behavior can be expected for similar cars with hatchback.

Cluster \#1 and \#2 are situated at the front of the car, whereas cluster \#3 and \#4 are located at the rear of the car. Therefore, these clusters have a strong influence on the rear backscatter. The monostatic scattering characteristics of the simplified scattering model for azimuth angle from $-50^{\circ}$ to $50^{\circ}$ is shown in Fig. 13. The model consists of only the clusters \#1 to \#4, both in vertical (left) and horizontal (right) polarization. The reference is the reflection coefficient obtained by the ray-tracing simulation of the Ford Focus model.

The virtual scattering center representation shows a good agreement with the ray-tracing simulation for the shown azimuth angles. At the sides of the car, the virtual scattering center representation does not match the original ray-tracing simulation, since the virtual scattering centers at the side of the car were not considered.

For 10 clusters, the comparison is shown in Fig. 14 for vertical (left) and horizontal (right) polarization.

Considering the first 10 clusters, the agreement between virtual scattering center representation and original ray-tracing simulation is very good for the whole azimuth angle.

This confirms the validity of the virtual scattering center representation with 10 clusters each with its own scattering characteristic.

\section{CONCLUSION}

In this paper, bistatic measurements and ray-tracing simulations have been compared to validate the ray-tracing simulations 
of a detailed car model. Monostatic simulations were used to determine the scattering centers. By forming clusters and representing them by virtual scattering centers with associated scattering characteristics led to a simple car model for monostatic simulations in the azimuth plane. This shows that ray-tracing simulations of complex objects allow the derivation of simplified scattering models. In general, the developed algorithm can be applied to arbitrary complex three-dimensional objects delivering simplified models with similar scattering characteristics. This is important, since only by reducing the complexity of large objects it becomes possible to simulate superior problems like automotive radar scenarios in realistic traffic situations. The herein presented monostatic model offers a proven base for such simulations. The presented method enables quick comparisons of different radar configurations. The extension to a bistatic model will make this method applicable to other problems and can be used to, e.g. simplify and improve channel estimation simulations for mobile communications.

\section{REFERENCES}

[1] J. Odendaal and P. Niemand, "Statistical properties of radar backscatter data for imaging applications," IEEE Trans. Instrum. Meas., vol. 51, pp. 670-672, Mar. 2003.

[2] K.-T. Kim, D.-K. Seo, and H.-T. Kim, "Radar target identification using one-dimensional scattering centres," Proc. Inst. Elect. Eng.-Radar, Sonar and Navigation, vol. 148, pp. 285-296, Oct. 2001.

[3] S. Chaudhuri and W.-M. Boerner, "A polarimetric model for the recovery of the high-frequency scattering centers from bistatic-monostatic scattering matrix data," IEEE Trans. Antennas Propag., vol. 35, pp. 87-93, Jan. 1987.

[4] H. Borrion, H. Griffiths, P. Tait, D. Money, and C. Baker, "Scattering centre extraction for extended targets," in Proc. IEEE Int. Radar Conf., May 2005, pp. 173-178.

[5] S. M. Rao, D. R. Wilton, and A. W. Glisson, "Electromagnetic scattering by surfaces of arbitrary shape," IEEE Trans. Antennas Propag., vol. 30, no. 3, pp. 409-418, May 1982.

[6] C. M. Furse, S. P. Mathur, and O. P. Gandhi, "Improvements to the finite-difference time-domain method for calculating the radar cross section of a perfectly conductig target," IEEE Trans. Microw. Theory Tech., vol. 38, no. 7, pp. 919-927, Jul. 1990.

[7] F. Weinmann, "Ray tracing with PO/PTD for RCS modeling of large complex objects," IEEE Trans. Geosci. Remote Sensing, vol. 54, pp. 1797-1806, Jun. 2006.

[8] A. Tzoulis and T. F. Eibert, "A hybrid FEBI-MLFMM-UTD method for numerical solutions of electromagnetic problems including arbitrarily shaped and electrically large objects," IEEE Trans. Antennas Propag., vol. 53, no. 10, pp. 3358-3366, Oct. 2005.

[9] M. Domingo, F. Rivas, J. Pérez, R. P. Torres, and M. F. Cátedra, "Computation of the RCS of complex bodies modeled using NURBS surfaces," IEEE Antennas Propag. Mag., vol. 37, no. 6, pp. 36-47, Dec. 1995.

[10] J. M. Rius, M. Ferrando, and L. Jofre, "GRECO: Graphical Electromagnetic Computing for RCS prediction in real time," IEEE Antennas Propag. Mag., vol. 35, no. 2, pp. 7-17, Apr. 1993.

[11] R. Bhalla and H. Ling, "Three-dimensional scattering center extraction using the shooting and bouncing ray technique," IEEE Trans. Geosci. Remote Sensing, vol. 44, pp. 1445-1453, Nov. 1996.

[12] J. Tsao and B. D. Steinberg, "Reduction of sidelobe and speckle artifacts in microwave imaging: The CLEAN technique," IEEE Trans. Antennas Propag., vol. 36, no. 4, pp. 543-556, Apr. 1988.

[13] L.-C. T. Chang, I. J. Gupta, W. D. Burnside, and C.-L. T. Chang, "A data compression technique for scattered fields from complex targets," IEEE Trans. Antennas Propag., vol. 45, no. 8, pp. 1245-1251, Aug. 1997.

[14] T. Fügen, J. Maurer, T. Kayser, and W. Wiesbeck, "Capability of 3D ray tracing for defining parameter sets for the specification of future mobile communications systems," IEEE Trans. Antennas Propag., vol. 54, no. 11, Nov. 2006.
[15] T. Fügen, J. Maurer, T. Kayser, and W. Wiesbeck, "Verification of $3 \mathrm{D}$ ray-tracing with non-directional and directional measurements in urban macrocellular environments," in Proc. 63rd IEEE Vehicular Technology Conf. VTC-2006 Spring, 2006, vol. 6, pp. 2661-2665.

[16] J. Maurer, "Strahlenoptisches Kanalmodell für die Fahrzeug-FahrzeugFunkkommunikation," Dissertation am , Institut für Höchstfrequenztechnik und Elektronik (IHE), Universität Karlsruhe (TH), Karls, Germany, Jul. 2005, 0942-2935.

[17] J. Maurer, T. Fügen, T. Schäfer, and W. Wiesbeck, "A new inter-vehicle communications (IVC) channel model," in Proc. 60th IEEE Veh. Technol. Conf. VTC-2004 Fall, 2004, vol. 1, pp. 9-13.

[18] R. Schneider, D. Didascalou, and W. Wiesbeck, "Impact of road surfaces on millimeter-wave propagation," IEEE Trans. Veh. Technol., vol. 49, pp. 1314-1320, Jul. 2000.

[19] M. Younis, J. Maurer, J. Fortuny-Guasch, R. Schneider, and W. Wiesbeck, "Interference from $24-\mathrm{GHz}$ automotive radars to passive microwave remote sensing satellites," IEEE Trans. Geosci. Remote Sensing, vol. 42, pp. 1387-1398, Jul. 2004.

[20] T. C. Cormen, Introduction to Algorithms, 1st ed. Cambridge, MA: MIT Press, 2001.

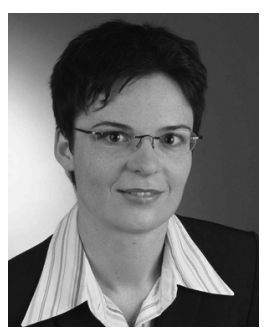

Karin Schuler was born in St. Georgen, Germany, in 1976. She received the DEA (M.S.E.E.) degree in 2002 from Ecole Nationale Supérieure d'Electronique et de Radioélectricité (ENSERG), Grenoble, France, and the Dipl.-Ing. and Ph.D. degrees from the Universität Karlsruhe (TH), Germany, in 2003 and 2007 , respectively.

In 2000, she spent six months as a Visiting Scientist at the National Oceanic and Atmospheric Administration (NOAA), Boulder, CO, where she worked on passive remote sensing. Afterwards, she was with the Institut für Höchstfrequenztechnik und Elektronik (IHE), Universität Karlsruhe (TH), Germany, as a Research Assistant. Her research areas have been focused on millimeter wave antennas, digital beamforming and automotive radar. Currently, she works for EADS Defence Electronics, Ulm, Germany.

Dr. Schuler won the 2003 EADS student award for her work on millimeter wave antennas and is coauthor of the paper winning the EEEfCOM Innovationspreis 2003 awarded by Rohde\&Schwarz, together with Gerotron $\mathrm{GmbH}$.

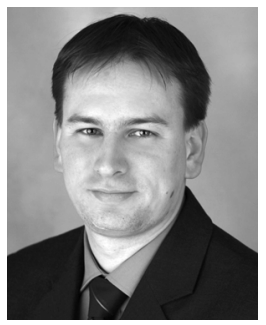

Denis Becker was born in Trier, Germany, in 1979. $\mathrm{He}$ studied electrical engineering and information technology at the Universitaet Karlsruhe (TH), Germany, where he received the Dipl.-Ing. (M.S.E.E.) degree in October 2006. He is currently working towards the Dr.-Ing. (Ph.D.E.E.) degree.

Since March 2007, he has been with the Institut fuer Hoechstfrequenztechnik und Elektronik (IHE), Universitaet Karlsruhe (TH), as a Research Associate. His research topics are focused on automotive radar and new digital beam forming signal processing techniques and concepts.

Mr. Becker won the Continental Auto-motivated Student Award 2005 and the EADS Defence Electronics ARGUS Award 2007 for his work on a novel DBF approach.

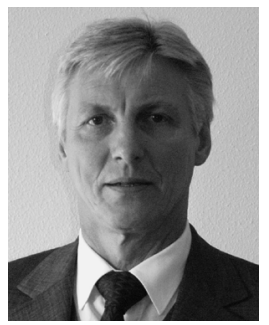

Werner Wiesbeck (SM'87-F'94) received the Dipl.Ing. (M.S.E.E.) and the Dr.-Ing. (Ph.D.E.E.) degrees from the Technical University Munich, in 1969 and 1972, respectively.

From 1972 to 1983, he was with AEG-Telefunken in various positions including that of head of $R \& D$ of the Microwave Division in Flensburg and marketing director Receiver and Direction Finder Division, Ulm. During this period he had product responsibility for mm-wave radars, receivers, direction finders and electronic warfare systems. From 1983 to 2007 he was Director of the Institut für Höchstfrequenztechnik und Elektronik (IHE) at the University of Karlsruhe (TH), where he had been Dean of the Faculty of Electrical Engineering and he is now Distinguished Scientist at the Karlsruhe Institute of Technology. Research topics include electromagnetics, antennas, wave propagation, communications, Radar and remote sensing. 
In 1989 and 1994, respectively, he spent a six months sabbatical at the Jet Propulsion Laboratory, Pasadena.

Dr. Wiesbeck is the recipient of a number of awards, including the IEEE Millennium Award, the IEEE GRS Distinguished Achievement Award, the Honorary Doctorate (Dr. h.c.) from the University Budapest/Hungary, the Honorary Doctorate (Dr.-Ing. E.h.) from the University Duisburg/Germany, and the IEEE Electromagnetics Award 2008. He is an Honorary Life Member of IEEE GRS-S, a Member of the Heidelberger Academy of Sciences and a Member of acatech (German Academy of Engineering and Technology). He is a member of the IEEE GRS-S AdCom (1992-2000), Chairman of the GRS-S Awards Committee (1994-1998, 2002-2007), Executive Vice President IEEE GRS-S (1998-1999), President IEEE GRS-S (2000-2001), Associate Editor of the IEEE TRANSACTIONS ON ANTENNAS AND PROPAGATION (1996-1999), past Treasurer of the IEEE German Section (1987-1996, 2003-2007). He has been General Chairman of the ' 88 Heinrich Hertz Centennial Symposium, the '93 Conference on Microwaves and Optics (MIOP '93), the Technical Chairman of International mm-Wave and Infrared Conference 2004, Chairman of the German Microwave Conference GeMIC 2006 and he has been a member of the scientific committees and TPCs of many conferences. He is a member of an Advisory Committee of the EU—Joint Research Centre (Ispra/Italy), and he is an advisor to the German Research Council (DFG), to the Federal German Ministry for Research (BMBF) and to industry in Germany. 